\title{
FATORES QUE INFLUENCIAM NA INTERRUPÇÃO DO ALEITAMENTO MATERNO EXCLUSIVO EM NUTRIZES
}

\section{FACTORS THAT INFLUENCE THE INTERRUPTION OF EXCLUSIVE BREASTFEEDING IN NURSING MOTHERS}

\author{
$\underline{\text { Manuela Carla de Souza Lima Daltro }}{ }^{1}, \underline{\text { Uany da Cruz Vale }}{ }^{2}, \underline{\text { Milena Nunes Alves de Sousa }}{ }^{3 *}$, \\ $\underline{\text { Bruna Alencar Castro }}{ }^{4}$, Larissa de Araújo Batista Suárez $^{5}, \&$ André Luiz Dantas Bezerra ${ }^{6}$ \\ ${ }^{1,4,6}$ Centro Universitário de Patos (UNIFIP); ${ }^{3,5,6}$ Faculdade São Francisco da Paraíba (FASP); \\ ${ }^{5}$ Universidade Católica de Pernambuco (UNICAP) \\ 1* manueladaltro@fiponline.edu.br ${ }^{2} \underline{\text { uanyvale@ hotmail.com }}{ }^{3} \underline{\text { milenanunes@ fiponline.edu.br }}{ }^{4} \underline{\text { alencar_@ @hotmail.com }}$ \\ ${ }^{5}$ labsuarez@gmail.com ${ }^{6}$ dr.andreldb@gmail.com
}

\section{ARTIGO INFO.}

Recebido em: 14.05.2021

Aprovado em: 19.08.2021

Disponibilizado em: 24.08.2021

\section{Palavras-CHaVe:}

Assistência Alimentar; Aleitamento Materno; Lactente. KEYWORDS:

Food Assistance; Breastfeeding; Infant.

*Autor Correspondente: Sousa, M. N. A., de

\section{RESUMO}

O aleitamento materno é o elemento fundamental para as crianças nos primeiros meses de vida, a fim de garantir o crescimento e o desenvolvimento psicológico e motor apropriado, atender as necessidades nutricionais da criança, propiciar fatores de promoção e proteção para a saúde materno-infantil. Além disso, também irá favorecer o vínculo mãe-filho quando o ato de amamentar é bem vivenciado pelas mães. Tratou-se de uma pesquisa de campo, descritiva, exploratória e quantitativa, objetivando avaliar os fatores que influenciam na interrupção do aleitamento materno exclusivo em nutrizes. A pesquisa foi realizada de forma online por meio de um questionário virtual. A população foi formada por todas as mães de crianças até 18 meses que estavam participando de um grupo de mães, na cidade de Patos, Paraíba. A amostragem foi constituída pelas primeiras 60 voluntárias que aceitaram participar, considerando tal amostragem equivalente a $100 \%$ do total proposto. A coleta de dados foi dada através da aplicação de um questionário semiestruturado contendo questões fechadas. Os dados da amostra foram analisados, tabulados e graficados utilizando o software Statistical Package for the Social Sciences (SSPSS). Os resultados mostram que os problemas que mais influenciaram as nutrizes quanto à interrupção do aleitamento foram fissuras no mamilo (55\%), ingurgitamento mamário $(30 \%)$ e baixa produção de leite $(30 \%)$. Importante mencionar que significativa parcela relatou dor durante a amamentação $(60 \%) \mathrm{e}$ apenas $17,6 \%$ relataram amamentação exclusiva só até os seis meses. Espera-se que a partir destas informações os profissionais de saúde exerçam o papel fundamental de ajudar as mães com as orientações corretas, fazendo com que ocorra o sucesso do aleitamento materno exclusivo.

\section{ABSTRACT}

Breastfeeding is the fundamental element for children in the first months of life, in order to guarantee growth and appropriate psychological and motor development, meet the child's nutritional needs, provide factors for the promotion and protection of maternal and child health. In addition, it will also provide the mother-child bond when the act of breastfeeding is well experienced by mothers. It was a descriptive, exploratory and quantitative field research, aiming to evaluate the factors that influence the interruption of exclusive breastfeeding in nursing mothers. The survey was performed online using a virtual questionnaire. The population was formed by all mothers of children up to 18 months of age who were participating of a group of mothers, in Patos, Paraíba. The sample consisted of the first 60 volunteers who agreed to participate, considering such sampling equivalent to $100 \%$ of the proposed total. Data collection was performed through the application of a semi-structured questionnaire containing closed questions. The sample data were analyzed, tabulated and graphed using the Statistical Package for the Social Sciences (SPSS) software. The results show that the problems that most influenced nursing women regarding the interruption of breastfeeding were nipple fissures (55\%), breast engorgement (30\%) and low milk production (30\%). It is important to mention that a significant proportion reported pain during breastfeeding (60\%) and only $17.6 \%$ reported exclusive breastfeeding only until six months. Based on this information, health professionals are expected to play a key role in helping mothers with the right orientation, making exclusive breastfeeding successful. 
Citação (APA): Daltro, M. C. de S. L., Vale, U. da C., Sousa, M. N. A., de, Castro, B. A., Suárez, L. de A. B., \& Bezerra, A. L. D. (2021).

Fatores que influenciam na interrupção do aleitamento materno exclusivo em nutrizes. Brazilian Journal of Production Engineering, 7(3), 153-162.

\section{INTRODUÇÃO}

O leite materno é o melhor alimento para criança até os seis meses de vida (Silva, Barreto, Bezerra, Bezerra, \& Bezerra, 2015) pois é apto a suprir, sozinho, as necessidades nutricionais da criança nos primeiros seis meses e permanece sendo uma importante fonte de nutrientes até o segundo ano de vida (Brasil, 2009a).

Sendo um alimento fundamental aos recém-nascidos, o leite materno possui 160 substâncias, é rico em proteínas, calorias, água, ferro, vitaminas, minerais, lipídios e lactose e ainda possui nutrientes importantes para que o bebê se desenvolva de forma saudável (Viana, 2017). Além disso, é livre de contaminações e possui fatores de proteção contra infecções, com destaque para a imunoglobulina A (IgA), Imunoglobulina M (IgM) e a Imunoglobulina G (IgG), macrófagos, neutrófilos, linfócitos B e T, lactoferrina, dentre outros (Amaral, 2016).

A amamentação é a forma mais eficiente para alimentar e proteger o recém-nascido e é entendida como um processo fisiológico que deve acontecer de forma natural e exclusivo até os seis meses de vida (Viana, 2017). A escolha de amamentar é um processo complexo, inspirado pelo desejo e encorajamento da mulher, pelas experiências positivas vividas dos seus familiares e amigas, e também pelo conhecimento e apoio sobre a amamentação (Primo et al., 2016).

$\mathrm{O}$ ato de amamentar é mais do que o caminho do leite de um organismo para outro, ele é um método de gerar um vínculo e interação mãe-bebê e por ocorrer esta comunicação, o bebê irá familiarizar-se com o mundo a sua volta (Primo et al., 2016). Onde irá promover o desenvolvimento facial infantil, ajudando na mastigação, deglutição, respiração e articulação dos fonemas, no que tange aos aspectos relacionados ao desenvolvimento sensório motor oral, principalmente no que se refere à posição, pega, força de sucção e coordenação entre as funções de sucção, deglutição e respiração (Silveira, Prade, Ruedell, Haeffner, \& Weinmann, 2013).

A amamentação possui diversas vantagens, pois o leite materno possui abundantes elementos, estimulando a imunidade contra as doenças alérgicas, a desnutrição, a obesidade, o câncer, as doenças cardiovasculares, digestivas e urinárias, dentre outros. Além disto, ocorre uma aproximação entre mãe e filho objetivando um vínculo afetivo (Brasil, 2009a). Ainda, favorece a saúde da mulher, prevenindo o câncer de mama, colaborando com a perda de peso, auxiliando a involução uterina, agindo como método contraceptivo e possibilitando uma aproximação entre mãe e filho, estreitando o vínculo afetivo (Brasil, 2009a; Escarce, Araújo, Friche, \& Motta, 2013).

Observa-se que mesmo com todos os benefícios vistos, ainda existem discordâncias quanto às taxas de Aleitamento Materno Exclusivo (AME) até o sexto mês de vida da criança. Segundo a II Pesquisa de Prevalência de Aleitamento Materno nas capitais brasileiras e Distrito Federal, estima-se que o aleitamento materno exclusivo possui tempo médio de 54,11 dias com predomínio de $41 \%$ nas capitais do Brasil, distante do que é sugerido. E, a Organização Mundial de Saúde, estima que se até 2025 os índices de aleitamento materno exclusivo 
chegassem até $50 \%$ nos países do mundo, impediria 823.000 mortes infantis por ano (Brasil, 2009b).

Brandão, Silva, Gouveia e Soares (2012) definem desmame precoce como a interrupção do aleitamento materno antes do período indicado pelas portarias ministeriais e introdução de outros alimentos na dieta da criança, com o objetivo de trocar ou complementar o leite materno quando ele deveria ser exclusivo. A interrupção do aleitamento é constante, e tem causado grandes riscos à saúde da criança, inclusive a morte de alguns recém-nascidos, pois, sem os fatores de proteção presentes no leite, elas ficam expostas a doenças e infecções graves, pois, o organismo infantil ainda é imaturo e não é capaz de se defender sozinho de graves ameaças.

Ademais, o desmame pode ser referente à primiparidade, baixo nível social, baixa escolaridade, falta de conhecimento sobre como amamentar, uso precoce de fórmulas, uso de chupetas, intercorrências com as mamas, hospitalização da criança, prematuridade e interferência familiar (Moreno \& Schmidt, 2014).

Diante do exposto, o objetivo deste trabalho foi avaliar os fatores que influenciam na interrupção do aleitamento materno exclusivo em nutrizes. $\mathrm{O}$ entendimento de tais elementos permite a elaboração de ações que possam estimular o aleitamento materno, corroborando com a promoção da saúde e o desenvolvimento infantil.

\section{Metodologia}

Tratou-se de uma pesquisa de campo, descritiva, exploratória e quantitativa. A pesquisa foi realizada de forma on-line por meio de um questionário virtual, com nutrizes do município de Patos, no Estado da Paraíba.

A população constituía-se de 60 mães de crianças com até 18 meses as quais estavam participando de um grupo de mães, na cidade de Patos no estado da Paraíba. Logo, 100\% do universo de pesquisa participaram voluntariamente do estudo. Apesar disto, foram adotados os seguintes critérios de inclusão: ser maior de dezoito anos e ter filho de até 18 meses. Estavam isentas de participar aquelas mulheres incapacitadas de responder o questionário por questões psíquicas.

A coleta de dados, realizada entre agosto e outubro de 2020, efetivou-se mediante aplicação de um questionário contendo questões fechadas, abordando os seguintes conteúdos: fatores sociais, demográficos e obstétricos, rede de apoio às lactantes, dados sobre aleitamento materno (conhecimento sobre amamentação, importância do aleitamento materno, dificuldades para realizar o aleitamento materno, identificação dos fatores que levaram ao desmame precoce) e tempo de amamentação exclusiva. $\mathrm{O}$ instrumento foi disponibilizado pelo grupo de WattsApp das mães que estavam no grupo anteriormente citado.

Como análise opinativa, os dados da amostra foram analisados, tabulados e graficados utilizando o software Statistical Package for the Social Sciences (SSPSS, versão 21.0). A realização deste estudo considerou a Resolução $n^{\circ}$ 510/16 e 580/18 do Conselho Nacional de Saúde que rege sobre a ética da pesquisa envolvendo seres humanos direta ou indiretamente,

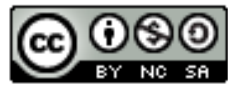



$153-162$.

em que assegurou a garantia de que a privacidade do sujeito da pesquisa será preservada. A pesquisa foi aprovada pelo Comitê de Ética em Pesquisa com Seres Humanos do Centro Universitário de Patos (UNIFIP), sob parecer de número 3. 849.910.

\section{Resultados}

\section{Fatores sociais, demográficos e obstétricos}

Foram incluídas no presente estudo 60 mães (100\% do universo de pesquisa). A idade das mães variou de 20 a 42 anos $(\mathrm{m}=30,12$ anos). Destas, $42(70 \%)$ possuíam nível superior, 48 (80\%) eram casadas, $41(68,3 \%)$ eram primíparas, 32 (53,3\%) amamentaram anteriormente, $48(80 \%)$ foram amamentadas pelas suas mães e $32(53,3 \%)$ relataram que as mulheres da sua família amamentaram. Além disso, 50 (83,3\%) tiveram gravidez sem risco, 100\% realizaram pré-natal, destas $32(53,3 \%)$ realizaram na rede privada (Tabela 1$)$.

Tabela 1. Fatores sociodemográficos e obstétricos de mães entrevistadas, na cidade de Patos-

PB.

\begin{tabular}{|c|c|c|}
\hline VARIÁVEIS & $\mathbf{N}=\mathbf{6 0}$ & $\%$ \\
\hline \multicolumn{3}{|l|}{ Escolaridade } \\
\hline Analfabeto & 0 & 0,0 \\
\hline Ensino Básico & 6 & 10 \\
\hline Ensino Secundário & 12 & 20 \\
\hline Ensino Superior & 42 & 70 \\
\hline \multicolumn{3}{|l|}{ Estado Civil } \\
\hline Solteira & 7 & 11,7 \\
\hline Casada & 48 & 80 \\
\hline União de fato & 4 & 6,7 \\
\hline Divorciada & 1 & 1,7 \\
\hline Viúva & 0 & 0,0 \\
\hline \multicolumn{3}{|l|}{$N^{0}$ de filhos } \\
\hline 01 & 41 & 68,3 \\
\hline 02 & 11 & 18,4 \\
\hline 03 & 8 & 13,3 \\
\hline \multicolumn{3}{|c|}{ Amamentou anteriormente? } \\
\hline $\operatorname{Sim}$ & 32 & 53,3 \\
\hline Não & 28 & 46,7 \\
\hline \multicolumn{3}{|c|}{ Sua mãe lhe amamentou? } \\
\hline $\operatorname{Sim}$ & 48 & 80 \\
\hline Não & 12 & 20 \\
\hline \multicolumn{3}{|c|}{ As mulheres da sua família amamentaram? } \\
\hline Sim & 32 & 53,3 \\
\hline Não & 1 & 1,7 \\
\hline Quase todas & 21 & 35 \\
\hline Nenhuma & 6 & 10 \\
\hline \multicolumn{3}{|l|}{ Gravidez } \\
\hline Normal & 50 & 83,3 \\
\hline De risco & 10 & 16,7 \\
\hline \multicolumn{3}{|l|}{ Realizou pré-natal? } \\
\hline Sim & 60 & 100,0 \\
\hline Não & 0 & 0,0 \\
\hline Parcial & 0 & 0,0 \\
\hline \multicolumn{3}{|l|}{ Onde? } \\
\hline Centro de saúde & 26 & 43,4 \\
\hline Privada & 32 & 53,3 \\
\hline Outro & 2 & 3,3 \\
\hline
\end{tabular}

Fonte: Autores (2020). 

153-162.

\section{Rede de apoio às lactantes}

Na tabela 2, nota-se que $48(80 \%)$ das mães relataram que tiveram ajuda na primeira mamada, sendo que $26(43,3 \%)$ foram assistidas por enfermeiros, 57 (95\%) tiveram ajuda no puerpério e destas, $32(53,3 \%)$ foram ajudadas pelo marido.

Tabela 2. Rede de apoio às mães entrevistadas, na cidade de Patos-PB.

\begin{tabular}{ccc}
\hline VARIÁ VEIS & n & $\%$ \\
\hline Teve ajuda na primeira mamada? & & \\
Sim & 48 & 80 \\
Não & 12 & 20 \\
Quem ajudou? & & \\
Enfermeiro & 26 & 43,3 \\
Médico & 4 & 6,7 \\
Familiar & 18 & 30,0 \\
Fisioterapeuta & 0 & 0 \\
Ninguém & 12 & 20,0 \\
Outros & 4 & 6,7 \\
Teve ajuda no puerpério? & & \\
Sim & 57 & 95 \\
Não & 3 & 5 \\
De quem? & & \\
Marido & 32 & 53,3 \\
Mãe & 20 & 31,5 \\
Ninguém & 3 & 5,0 \\
Outros & 5 & 8,3 \\
\hline Fonte: Autores & &
\end{tabular}

\section{Aleitamento Materno}

Fonte: Autores (2020).

$50 \%(83,3 \%)$ relataram ter tido informações sobre o aleitamento materno, em que $19(38,8 \%)$ tiveram informações através de consultas privadas, 18 (33,3\%) foram informadas pelos enfermeiros e $49(90,7 \%)$ receberam informações que os benefícios da amamentação são tanto para o bebê quanto para a mãe.

Ainda, 73,3\% (n=44) das mães ainda amamentavam, 17,6\% $(n=6)$ relataram que seu filho deixou de mamar com seis meses, $31(51,7 \%)$ só davam leite materno exclusivo aos seus filhos, $58(96,7 \%)$ iniciaram o aleitamento materno no hospital e $45(75 \%)$ iniciaram o aleitamento na $1^{\mathrm{a}}$ hora de vida do bebê.

Os problemas mais apresentados pelas gestantes foram: fissuras no mamilo (55\%), ingurgitamento mamário (30\%) e baixa produção de leite (30\%). $36(60,0 \%)$ relataram dor nas primeiras mamadas e $32(53,3 \%)$ afirmaram que a dor surgia quando o bebê iniciava a mamada (Tabela 3).

Tabela 3. Características da amamentação e percepção das mães entrevistadas, na cidade de Patos-PB.

\begin{tabular}{lcc}
\hline VARIÁVEIS & $\mathbf{N = 6 0}$ & $\%$ \\
\hline Foi informada sobre o aleitamento durante a gravidez? & 50 & 83,3 \\
Sim & 10 & 16,7 \\
Não & & \\
Onde? & 18 & 30,0 \\
Centro de saúde & 19 & 31,7 \\
Privada & 6 & 10,0 \\
Hospital & 6 & 10,0 \\
Curso de preparação para o parto & 10 & 16,7 \\
Sem informação & & \\
\hline
\end{tabular}



$153-162$.

\begin{tabular}{|c|c|c|}
\hline \multicolumn{3}{|l|}{ Quem informou sobre a amamentação? } \\
\hline Enfermeiro & 18 & 33,3 \\
\hline Médico de família & 0 & 0,0 \\
\hline Pediatra & 1 & 1,9 \\
\hline Obstetra & 16 & 29,6 \\
\hline Fisioterapeuta & 5 & 9,3 \\
\hline Familiar e amigos & 7 & 13 \\
\hline Livros e revistas & 1 & 1,9 \\
\hline Outro & 6 & 11,1 \\
\hline \multicolumn{3}{|l|}{ As informações foram sobre as vantagens para: } \\
\hline Mãe & 7 & 11,7 \\
\hline Pai & 4 & 6,7 \\
\hline Bebê & 49 & 81,6 \\
\hline \multicolumn{3}{|l|}{ Quando deve ser iniciada a primeira amamentação? } \\
\hline Dentro da $1^{\text {a }}$ hora de vida logo que a mãe e o bebê estejam & 55 & 90 \\
\hline prontos & 4 & 6,7 \\
\hline Depois da $1^{\text {a }}$ hora de vida & 1 & 1,7 \\
\hline \multicolumn{3}{|l|}{ A hora do início não é importante } \\
\hline \multicolumn{3}{|l|}{ Qual a duração adequada da amamentação exclusiva? } \\
\hline 6 meses & 54 & 90 \\
\hline Até o bebê querer & 2 & 3,3 \\
\hline Enquanto tiver leite & 4 & 6,7 \\
\hline Não sei & 0 & 0,0 \\
\hline \multicolumn{3}{|l|}{ Qual a duração adequada para dar leite e outros } \\
\hline alimentos? & 16 & 26,7 \\
\hline 6 meses & 8 & 13,3 \\
\hline Até o bebê querer & 2 & 3,3 \\
\hline Enquanto tiver leite & 30 & 50 \\
\hline Enquanto for satisfatório para a mãe, bebê e pai & 4 & 6,7 \\
\hline \multicolumn{3}{|l|}{ Não sei } \\
\hline \multicolumn{3}{|l|}{ A amamentação é: } \\
\hline Exclusiva & 31 & 51,7 \\
\hline \multirow{2}{*}{\multicolumn{3}{|c|}{ Iniciou o aleitamento na maternidade? }} \\
\hline & & \\
\hline Sim & 58 & 96,7 \\
\hline Não & 2 & 3,3 \\
\hline \multicolumn{3}{|l|}{ Quando amamentou a $1^{\circ}$ vez? } \\
\hline Durante a $1^{a}$ hora de vida do bebê & 45 & 75 \\
\hline Depois da $1^{\mathrm{a}}$ até à $6^{\mathrm{a}}$ hora & 10 & 16,7 \\
\hline Depois da $6^{\mathrm{a}}$ hora de vida & 5 & 8,3 \\
\hline \multicolumn{3}{|l|}{ Problemas durante a amamentação* } \\
\hline Fissuras & 33 & 55 \\
\hline Ingurgitamento & 18 & 30 \\
\hline Bloqueio dos ductos & 12 & 20 \\
\hline Mastite & 4 & 6,7 \\
\hline Abscesso mamário & 3 & 5 \\
\hline Baixa produção & 18 & 30 \\
\hline \multicolumn{3}{|l|}{ Sentiu dor nas primeiras mamadas? } \\
\hline Sim & 36 & 60,0 \\
\hline Não & 24 & 40,0 \\
\hline \multicolumn{3}{|l|}{ Essa dor começava: } \\
\hline Quando o bebê iniciava a mamada & 32 & 53,3 \\
\hline Durante a mamada & 3 & 5,0 \\
\hline No fim da mamada & 0 & 0,0 \\
\hline Mantinha-se no intervalo das mamadas & 1 & 1,7 \\
\hline Sem dor & 24 & 40,0 \\
\hline
\end{tabular}

*Nota: algumas questões permitiram mais de uma resposta.

Fonte: Autores (2020). 
Citação (APA): Daltro, M. C. de S. L., Vale, U. da C., Sousa, M. N. A., de, Castro, B. A., Suárez, L. de A. B., \& Bezerra, A. L. D. (2021). Fatores que influenciam na interrupção do aleitamento materno exclusivo em nutrizes. Brazilian Journal of Production Engineering, 7(3), $153-162$.

\section{Tempo de amamentação exclusiva}

Somente 17,6\% das mães que participaram da pesquisa relataram que fizeram a amamentação exclusiva só até os seis meses e 5,9\% relataram que fizeram a amamentação até um ano e um mês (Gráfico 1). Importante frisar que 52,6\% não fizeram amamentação exclusiva, e já nos meses iniciais usavam fórmulas, promovendo o desmame precoce.

Gráfico 1. Tempo de amamentação

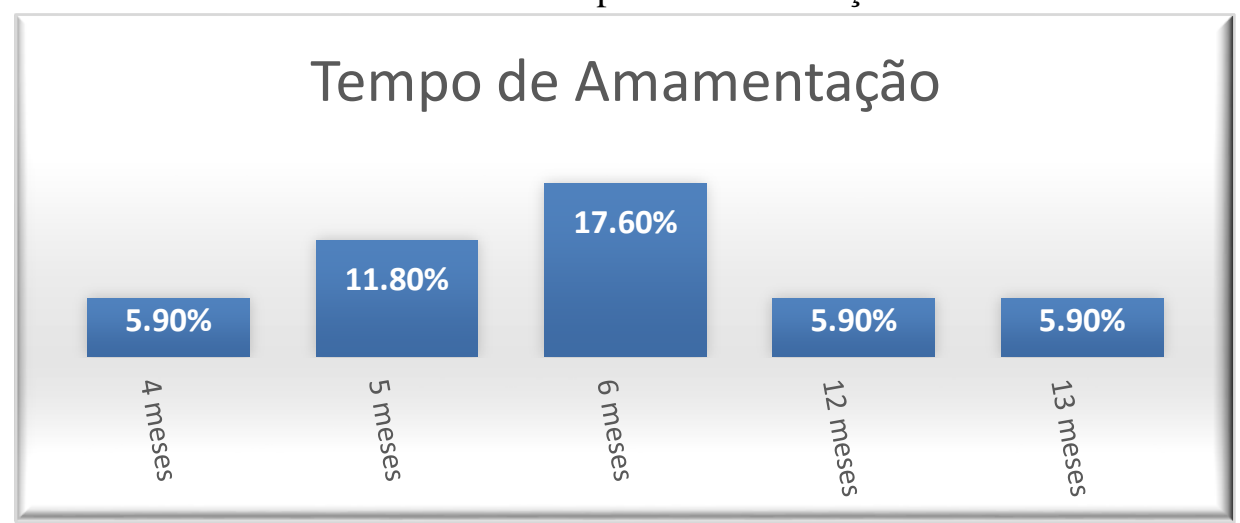

Fonte: Autores (2020).

\section{Discussão}

A maioria das mães possuía ensino superior, o que pode indicar um fator positivo em relação ao aleitamento, pois faz com que aumente a duração da amamentação e a compreensão de informações sobre a amamentação. Oliveira e Marques (2011) afirmam que mães com mais anos de estudos absorvem mais informações e estão mais propensas a aplicá-las em seu dia a dia.

O estado civil casado parecer incidir sobre ações protetivas. Alves, Oliveira e Rito (2018) em estudo intitulado "orientações sobre amamentação na atenção básica de saúde e associação com o aleitamento materno exclusivo" identificaram predomínio de mães casadas e ressaltaram que não possuir companheiro teve relação com o desmame precoce. Os autores ainda afirmaram a importância do apoio do parceiro para a mulher, especialmente quanto à amamentação exclusiva, pois seu estímulo é o mais significativo para que a mulher possa continuar amamentando.

1/5 das mães não haviam sido amamentadas, o que indica que o fator cultural pode influenciar negativamente o período da amamentação. Capucho, Forechi, Lima, Massaroni e Primo, (2017) abordam que as pessoas presentes no cotidiano da mãe estão diretamente ligadas ao processo de amamentação, ou seja, suas falas favorecem ou não a prática.

Relacionando-se à assistência pré-natal, $100 \%$ da amostra relataram ter realizado. Dado semelhante foi identificado por Kantovisck e Giustina (2016), cujo objetivo foi mostrar a importância da assistência ao pré-natal, proporcionando educação e promoção da saúde, além de esclarecer dúvidas e questionamentos das genitoras. Apesar do achado, foi encontrado que grande parte das mães estava realizando o acompanhamento de seu bebê em rede privada, o que difere do encontrado na pesquisa de Pereira, Oliveira, Tavares e Brito (2010), que a maioria das mães $(48 \%)$ fizeram o acompanhamento em unidade de pequeno porte, como postos de saúde.

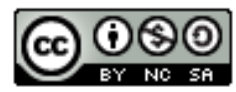


Mais de 1/3 da amostra relataram ter tido informações sobre o aleitamento materno. Martins e Zanatta (2007) afirmaram a importância de discutir com as gestantes as vantagens do aleitamento materno e as desvantagens do desmame ou introdução de alimentos complementares orientando também quanto à fisiologia da lactação reduzindo dessa forma, as dúvidas e ansiedades da mulher.

Ademais, o profissional da área da enfermagem foi quem mais orientou as mulheres sobre o ato de amamentar. Achado semelhante ao estudo de Shimoda, Aragaki, Sousa e Silva, (2014), os quais evidenciaram que se deve ao fato de o enfermeiro ser o profissional mais próximo da mulher durante o ciclo gravídico-puerperal, desempenhando um papel relevante nos programas de educação em saúde, durante o pré-natal, preparando a gestante para o aleitamento, para que no pós-parto o processo de adaptação seja facilitado e tranquilo, evitando dúvidas, dificuldades e possíveis complicações.

Quanto aos problemas que levaram ao desmame precoce, destacam-se fissuras, ingurgitamento e baixa produção de leite. Estes fatores assemelharam-se a pesquisa de Dos Santos, Resende, Maia, de Jesus Carvalho e Júnior (2020), que relataram como fator principal das mães realizarem o desmame precoce as fissuras mamilares.

Ainda sobre a temática acima, a pesquisa realizada por Alvarenga et al. (2017) diferiu do resultado, mostrando que os problemas mais significativos foram o uso da chupeta e o leite fraco. No entanto, todos esses fatores são utilizados pelas nutrizes em diversas culturas para explicar o abandono da amamentação, porém, sabe-se que o leite materno é ideal e são pouco frequentes as intercorrências que inviabilizam a amamentação.

Por conseguinte, mais da metade das puérperas mencionaram sentir dor durante a amamentação, uma grande maioria destas afirmaram sentir a dor no início da mamada. Logo, corroborando com tais achados, mães com trauma mamilar relataram significativamente maior nível de dor e interferência na amamentação, e constatou-se que maior intensidade e duração da dor teve maior influência com a atividade geral, o humor e o sono (Moimaz, Rocha, Garbin, \& Saliba, 2011). Outro estudo também realizado em São Paulo com 60 puérperas verificou que a persistência de lesão de mamilos está associada à dor mamilar (Shimoda et al., 2014).

O desmame precoce também foi evidenciado de forma crítica nesta pesquisa, uma vez que mais da metade não fiz amamentação exclusiva e já nos meses iniciais usavam fórmulas. Silva et al., (2018) verificaram que 35\% das mães de uma unidade básica de saúde de Pombal-PB desmamou seus filhos com um mês de vida. Este resultado é bem crítico, especialmente ao se conhecer os benefícios da amamentação exclusiva para o binômio mãe-filho.

Quanto às limitações do estudo, podem-se citar o tamanho da amostra e forma de coleta de dados, os quais poderiam ter sido contatadas outras mulheres que não faziam parte do grupo de mães da pesquisa.

\section{Conclusão}

O leite materno é o alimento ideal para o bebê durante o seu primeiro ano de vida, pois é através dele que o lactente estará bem nutrido na fase inicial da sua vida e protege de patologias futuras.

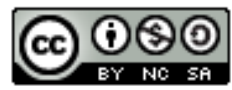


Citação (APA): Daltro, M. C. de S. L., Vale, U. da C., Sousa, M. N. A., de, Castro, B. A., Suárez, L. de A. B., \& Bezerra, A. L. D. (2021). Fatores que influenciam na interrupção do aleitamento materno exclusivo em nutrizes. Brazilian Journal of Production Engineering, 7(3), $153-162$.

Deste modo, o trabalho apontou que as puérperas sabem da importância do aleitamento materno, do seu tempo de duração ideal e dos seus benefícios. Principalmente aquelas mães que tinham o ensino superior, pois compreendem mais sobre o assunto e fazem o uso das informações. As mães que têm o apoio de um companheiro são mais propensas de amamentar, por motivos deles exercerem uma influência positiva na duração do aleitamento materno.

Concluiu-se, também, que grande parte das mães teve acesso a informações quanto ao aleitamento e o desmame, durante o pré-natal ou após o parto, onde é nesse tempo que surgem as dúvidas e as ansiedades maternas que podem ser fatores para o insucesso da amamentação, como as fissuras mamilares, o ingurgitamento mamário e o leite insuficiente, fazendo com que façam a introdução de fórmulas.

Espera-se que os profissionais de saúde exerçam o papel fundamental de ajudar as mães com as orientações, fazendo com que ocorra o sucesso do aleitamento materno exclusivo. Desta forma, quanto mais orientações as puérperas recebam sobre a importância do aleitamento, suas vantagens e seus benefícios para ambos, fazendo com que tenha um resultado positivo no vínculo afetivo entre a mãe e o bebê.

\section{Referências}

Alvarenga, S. C., de Castro, D. S., Leite, F. M. C., Brandão, M. A. G., Zandonade, E., \& Primo, C. C. (2017). Fatores que influenciam o desmame precoce. Aquichan, 17(1), 93-103. Recuperado de https://www.redalyc.org/pdf/741/74149923009.pdf

Alves, J. D. S., Oliveira, M. I. C. D., \& Rito, R. V. V. F. (2018). Orientações sobre amamentação na atenção básica de saúde e associação com o aleitamento materno exclusivo. Ciência \& Saúde Coletiva, 23, 1077-1088. https://doi.org/10.1590/1413$\underline{81232018234.10752016}$

Amaral, R. C. (2016). Fatores que contribuem para o desmame precoce e atuação da enfermagem. FACIDER-Revista Científica, 9(9), 1-17.

Brandão, E. C., da Silva, G. R. F., de Oliveira Gouveia, M. T., \& Soares, L. S. (2012). Caracterização da comunicação no aconselhamento em amamentação. Revista Eletrônica de Enfermagem, 14(2), 355-65. https://doi.org/10.5216/ree.v14i2.12748

Brasil. (2009a). Ministério da Saúde. Secretaria de Atenção à Saúde. Departamento de Atenção Básica. Saúde da criança: Nutrição infantil: aleitamento materno e alimentação. Brasília: Editora do Ministério da Saúde. Recuperado de https://bvsms.saude.gov.br/bvs/publicacoes/saude_crianca_nutricao_aleitamento_alimentacao .pdf

Brasil. (2009b). Ministério da Saúde. Secretaria de Atenção à Saúde. Departamento de Ações Programáticas e Estratégicas. II Pesquisa de prevalência de aleitamento materno nas capitais brasileiras e Distrito Federal. Brasília: Ministério da Saúde. Recuperado de https://portaldeboaspraticas.iff.fiocruz.br/biblioteca/ii-pesquisa-de-prevalencia-de-

aleitamento-materno-nas-capitais-brasileiras-e-distrito-federal/

Capucho, L. B., Forechi, L., Lima, R. D. C. D., Massaroni, L., \& Primo, C. C. (2017). Fatores que interferem na amamentação exclusiva. Revista Brasileira de Pesquisa em Saúde/Brazilian Journal of Health Research, 19(1), 108-113. Recuperado de https://periodicos.ufes.br/rbps/article/view/17725 
Citação (APA): Daltro, M. C. de S. L., Vale, U. da C., Sousa, M. N. A., de, Castro, B. A., Suárez, L. de A. B., \& Bezerra, A. L. D. (2021). Fatores que influenciam na interrupção do aleitamento materno exclusivo em nutrizes. Brazilian Journal of Production Engineering, 7(3), 153-162.

Dos Santos, A. A., Resende, M. A., Maia, G. P., de Jesus Carvalho, N. C., \& Júnior, A. D. P. F. (2020). O papel do enfermeiro na prevenção do desmame precoce. Revista Eletrônica Acervo Enfermagem, 2, e2232-e2232.

Escarce, A. G., de Araújo, N. G., de Lima Friche, A. A., \& Motta, A. R. (2013). Influence of guidance about breastfeeding in the behavior of a university hospital users. Revista CEFAC, 15(6), 1570-1582. https://doi.org/10.1590/S1516-18462013000600020

Kantovisck, M., \& Giustina, A. P. (2016). A importância da assistência no pré-natal. Recuperado de http://www.uniedu.sed.sc.gov.br/wp-content/uploads/2016/10/MARINESNEVES-KANTOVISCK..pdf

Martins, D. R., \& Zanatta, E. A. (2006). Percepções das mães acerca do aleitamento materno e desmame precoce. Revista de Enfermagem, 2 (2 e 3), 53-74.

Moimaz, S. A. S., Rocha, N. B., Garbin, A. J. I., \& Saliba, O. (2011). Relação entre aleitamento materno e hábitos de sucção não nutritivos. Ciência \& Saúde Coletiva, 16, $2477-$ 2484. https://www.scielo.br/pdf/csc/v16n5/a17v16n5.pdf

Moreno, P. D. F. B. B., \& Schmidt, K. T. (2014). Aleitamento materno e fatores relacionados ao desmame precoce. Cogitare Enfermagem, 19(3). 576-81.

Oliveira, K. M. P. de \& Marques, I. R. (2011) Situação do aleitamento materno no Brasil: uma revisão. Revista de Enfermagem Unisa,12(1), 73-78. Recuperado de https://docplayer.com.br/15189728-Situacao-do-aleitamento-materno-no-brasil-umarevisao.html

Pereira, R. S. V., Oliveira, M. I. C. D., Andrade, C. L. T. D., \& Brito, A. D. S. (2010). Fatores associados ao aleitamento materno exclusivo: o papel do cuidado na atenção básica. Cadernos de Saúde Pública, 26, 2343-2354. https://doi.org/10.1590/S0102-311X2010001200013

Primo C., Nunes C.O, Lima, B.F.A., Leite, E.M.C., Pontes, F.B.M, \& Brandão G., M.A. (2016). Quais fatores influenciam as mulheres na decisão de amamentar? Investigación y Educación en Enfermería, 34(1), 198-217. https://doi.org/10.17533/udea.iee.v34n1a22

Shimoda, G. T., Aragaki, I. M. M., Sousa, C. A. de, \& Silva, I. A. (2014). Associação entre persistência de lesão de mamilos e condições de aleitamento materno. REME: Revista Mineira de Enfermagem, 18, 68-74. https://doi.org/10.5935/1415-2762.20140006

Silva, A. M. D., Santos, M. C. S. D., Silva, S. R. D. M., Ferreira, F. Â., Freitas, R. D. S. C., Santos, R. E. A. D., \& Gouveia, M. T. (2018). Aleitamento materno exclusivo: empecilhos apresentados por primíparas. Rev. Enferm. UFPE on line, 12(12), 3205-3211. Recuperado de https://pesquisa.bvsalud.org/portal/resource/pt/biblio-999669

Silva, R. A., Barreto, C. C. M., Bezerra, A. M. F., Bezerra, K. K. S., \& Bezerra, W. K. T. (2015). Aleitamento materno: fatores que influenciam o desmame precoce. Revista Brasileira de Educação e Saúde,5(3), 01-07. Recuperado de http://oaji.net/articles/2016/26281461602690.pdf

Silveira, L. M. D., Prade, L. S., Ruedell, A. M., Haeffner, L. S. B., \& Weinmann, A. R. M. (2013). Influence of breastfeeding on children's oral skills. Revista de saúde pública, 47, $37-$ 43. https://www.researchgate.net/publication/236908109_Influence_of_breastfeeding_on_childre n's oral skills

Viana, M. A. (2007) A importância do aleitamento materno exclusivo. Trabalho de conclusão de curso - Faculdade de Ciências da Educação e Saúde. Brasília. Recuperado de https://repositorio.uniceub.br/jspui/bitstream/235/11737/1/21313612.pdf 